\title{
Electron Beam-Induced Charging and Modifications of Thin Films
}

Marek Malac ${ }^{1,2}$, Marco Beleggia ${ }^{5,7}$, Teddy Rowan ${ }^{1,3}$, Ray Egerton ${ }^{1,2}$, Masahiro Kawasaki ${ }^{4}$, Yoshio Okura ${ }^{4}$, Robert A. McLeod ${ }^{6}$

${ }^{1}$ National Institute of Nanotechnology, 11421 Saskatchewan Drive, Edmonton, Canada

2 Department of Physics, University of Alberta, T6G 2E1, Edmonton, Canada

${ }^{3}$ University of British Columbia. 2329 West Mall, Vancouver, V6T 1Z4, Canada

4 JEOL Ltd. 1-2 Musashino 3 chome, Akishima, Tokyo 198-8558, Japan

${ }^{5}$ Centre of Electron Nanoscopy, Denmark Technical University, Denmark

${ }^{6}$ Fondation Nanosciences, 23 rue des Martyrs, 38000 Grenoble, France

${ }^{7}$ Helmholtz-Zentrum Berlin fuer Materialen und Energie, 14109 Berlin, Germany

A thin film irradiated by high-energy primary electrons (PE) emits secondary electrons (SE). The SE are either emitted from the sample (SE $\alpha$ ) or travel within the sample (SE $\beta$ ) [1]. Figure 1 illustrates the various types of SEs. The PEs, SE $\alpha$ and the SE $\beta$ affect, by modifying the film properties, the rate at which the irradiated sample area reaches a steady state. Both PE and SE $\beta$ can cause radiation damage [2] and electron beam induced conductivity (EBIC) [1]. The discussed phenomena are relevant to sample charging, damage and to various implementations of hole-free phase plate (HFPP) [3, 4]. The beam-induced modifications can result in a spatiallydependent surface potential $\sigma(r)$, generating an electric field $E_{V}(r, z)$ that extends in the vacuum surrounding the sample [3].

An irradiated thin film tends toward a steady state, as indicated by the time dependence of SE $\alpha$ current and the evelution of the contrast transfer function (CTF) [3,4]. Figure 2 shows SE $\alpha$ signal (a) and, (b), the position of extrema in a CTF as a function of time. Both SE $\alpha$ and CTF time evolution can be fitted to:

$$
Y(t)=Y_{0}+A_{0} \exp \left(-t / t_{0}\right)
$$

Here $Y(t)$ and $Y_{0}$ are the dependent variable (SEa signal or CTF extrema position) and their initial values respectively, and $t_{0}$ is the exponential characteristic time, which we take as a measure of the settling time. The values of $t_{0}$ obtained from SE $\alpha$ and CTF correlate; they are a few seconds to a few tens of seconds for all studied materials and conditions. According to Eq. (1), the film state is within $2 \%$ of its asymptotic steady state after $t_{\mathrm{S}}=4 t_{0}$. A steady state is achieved when a balance is reached between the SE $\alpha$ leaving the sample and the current from ground electrode $I_{\mathrm{G}}$. At steady state, the electric field outside sample $E_{V}(r, z)$ reduces the $S E \alpha$ until it is fully compensated by the $I_{\mathrm{G}}$. When multiple processes control the settling the Eq. (1) includes more than one term. The settling time $t_{\mathrm{S}}$ is then determined by the slowest process. The $A_{0}>0$ for SE $\alpha$ decay and for Thon rings that decrease diameter with time (i.e. the defocus and charging term in a CTF contribute phase shift with the same sign). The $A_{0}<0$ for Thon rings when defocus and charging contribute opposite sign of phase shift. 
The irradiated area appears to remain close to the steady state after the PE beam is switched off $[3,4,5]$. This can be explained by existence of a charge-trapping layer on the film surface with electron tunneling governing the recombination charge transport. At steady state, the emitted SE $\alpha$ current density $j_{\mathrm{SE}}(r)$ equals to a tunneling current $j_{\mathrm{T}}(r)$ at every location $r$ of the film, as graphically depicted in Figure 3 for Fowler-Nordheim (FN) and Poole-Frenkel (PF) tunneling mechanisms. The tunneling current exponentially depends on electric field $\mathrm{E}_{\mathrm{T}}(r, z)$ inside the charge trapping layer which is related to the $j_{\mathrm{SE}}(r)$. When the PE irradiation ceases and the $j_{\mathrm{SE}}(r)$ goes to zero, an exponentially small decrease in $\mathrm{E}_{\mathrm{T}}(r, z)$ switches off the $j_{\mathrm{T}}(r)$ effectively maintaining the steady state charge distribution. Figure 3 indicates that both FN and PF mechanisms achieve steady state for moderate surface potential values.

The SE $\alpha$ escape depth $\delta$ is an important parameter in models capturing the behavior of irradiated films. Figure 4 shows the dependence of SE $\alpha$ signal and sample thickness for an insitu drilled amorphous carbon film at $600^{\circ} \mathrm{C}$. Because the $\mathrm{SE} \alpha$ escape from both surfaces, the $\delta$ must be less than $1 / 2(6.8 \mathrm{~nm})=3.4 \mathrm{~nm}$. Since the escape depth is large in insulators and small in conductors [1], amorphous carbon at $\mathrm{T}=600^{\circ} \mathrm{C}$ behaves as a good conductor.

The relative importance of EBIC, SE $\alpha$ emission and charge tunneling $j_{\mathrm{T}}(r)$ is determined by mobility of charge carriers in the film, PE current density, by SE yield and its dependence on bias and thickness and dielectric properties of any surface layers on the film including contamination. The contribution of SE $\beta$ to EBIC generation is small compared to PE [2]. 


\section{References}

[1] L. Reimer, Scanning Electron Microscopy $2^{\text {nd }}$ ed., Springer, 1998.

[2] RF Egerton, M Malac Microscopy and Microanalysis 10 (S02) (2004), p.1382.

[3] M. Malac et. al., Ultramicroscopy 118 (2012), p. 77.

[4] R. Danev et. al. PNAS, 111 (2015), p. 15635.

[5] J. Berriman and K. L. Leonard, Ultramicroscopy 19 (1986), p. 349.

[6] Support of NINT, NSERC, JEOL USA Inc. and JEOL Ltd. is gratefully acknowledged.

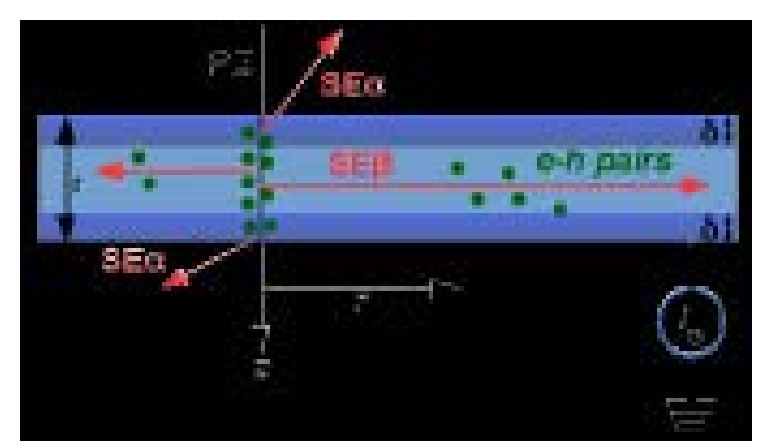

Fig. 1. Primary electrons (PE) incident on a thin film generates $\mathrm{SE} \alpha$ that escape to vacuum from a depth $\delta$, and SE $\beta$ that travel within the specimen. The PE and $\operatorname{SE} \beta$ generate $e$ - $h$ pairs that affect sample conductivity.
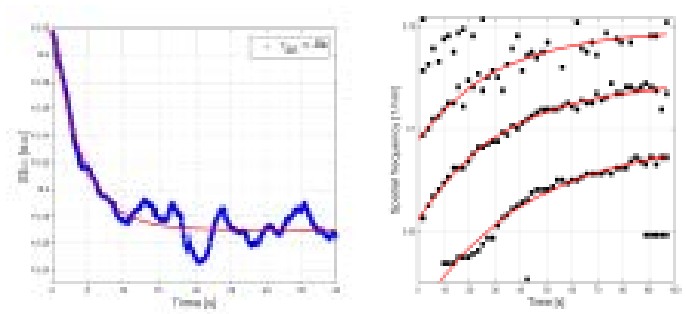

Fig. 2. Time traces of SE $\alpha$ current and CTF extrema from a $15 \mathrm{~nm}$ thick carbon film at room temperature and PE beam current density $j_{\mathrm{PE}}=0.1 \mathrm{~A} / \mathrm{cm}^{2}$. Both the SE $\alpha$ time traces and the position of extrema in CTF obey an exponential decay in Eq. (1): $\tau_{\mathrm{SE}} \sim 4 \mathrm{~s}, \tau_{\mathrm{CTF}} \sim 8$ to $10 \mathrm{~s}$.

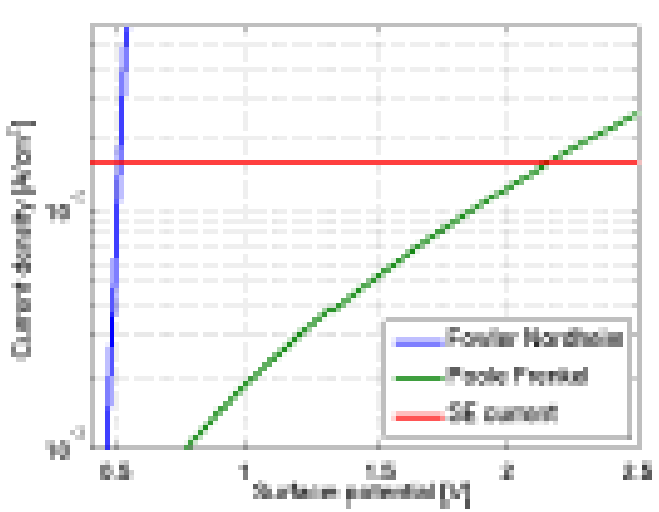

Fig. 3. At a steady state a balance between $j_{\mathrm{SE}}(r)$ and $j_{\mathrm{T}}(r)$ is maintained. An intersection of I-V curves corresponds to a balance. The FN tunneling (blue) has much steeper I$\mathrm{V}$ dependence than the PF tunneling (green). The $j_{\mathrm{SE}}(r)$ (red) is flat at typical values of surface potential.

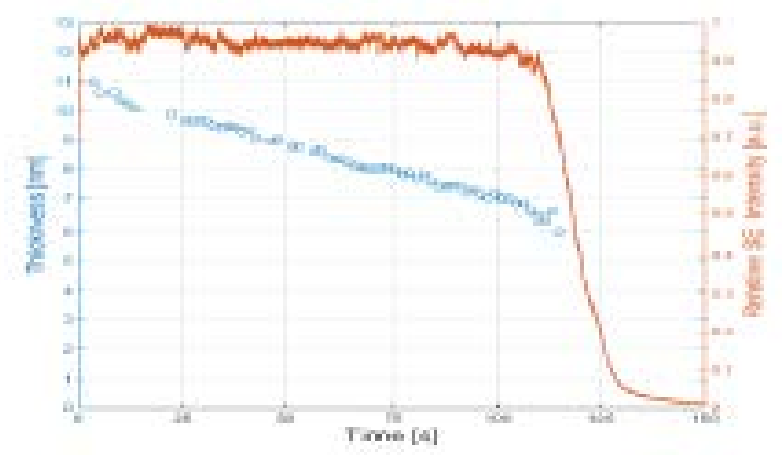

Fig. 4. SE $\alpha$ current and film thickness of an in-situ drilled carbon film at $600^{\circ} \mathrm{C}$. The SEa escape depth is less than $1 / 26.8 \mathrm{~nm}$, i.e. less than $\sim 30$ atomic layers. The $j_{\mathrm{PE}}=0.5 \mathrm{~A} / \mathrm{cm}^{2}$. 
